\title{
Implementing Tactile and Proximity Sensing for Crack Detection
}

\author{
Francesca Palermo ${ }^{1 \dagger}$, Jelizaveta Konstantinova ${ }^{1,3}$, Kaspar Althoefer ${ }^{1}$, \\ Stefan Poslad ${ }^{1}$, Ildar Farkhatdinov ${ }^{1,2 \dagger}$
}

\begin{abstract}
Remote characterisation of the environment during physical robot-environment interaction is an important task commonly accomplished in telerobotics. This paper demonstrates how tactile and proximity sensing can be efficiently used to perform automatic crack detection. A custom-designed integrated tactile and proximity sensor is implemented. It measures the deformation of its body when interacting with the physical environment and distance to the environment's objects with the help of fibre optics. This sensor was used to slide across different surfaces and the data recorded during the experiments was used to detect and classify cracks, bumps and undulations. The proposed method uses machine learning techniques (mean absolute value as feature and random forest as classifier) to detect cracks and determine their width. An average crack detection accuracy of $86.46 \%$ and width classification accuracy of $57.30 \%$ is achieved. Kruskal-Wallis results $(\mathbf{p}<\mathbf{0 . 0 0 1})$ indicate statistically significant differences among results obtained when analysing only force data, only proximity data and both force and proximity data. In contrast to previous techniques, which mainly rely on visual modality, the proposed approach based on optical fibres is suitable for operation in extreme environments, such as nuclear facilities in which nuclear radiation may damage the electronic components of video cameras.
\end{abstract}

\section{INTRODUCTION}

Background. Most of commercially exploited humanmachine interfaces for telerobotics rely on visual information to collect information on the remote environment [1], [2] which can be insufficient in complex, dynamic and unstructured environments with limited luminosity [3], [4]. It is especially difficult to perform surface and object material characterisation when only visual feedback is available. Hence, the introduction of a remote touch feedback modality becomes of great importance. Tactile feedback can provide important information about the remote environment and substantially improve the efficiency and safety of telerobotics tasks. Remote materials and objects characterisation is a typical application in telerobotics: remote control of mobile robots [5], [6], surgical robotics and training [7], nuclear waste management and remote material handling [8], [9]. An important task often performed in remote hazardous environments is the detection and characterisation of mechanical fractures of objects such as containers, tanks, pipes and other systems used for storing and processing chemical and radioactive waste. A surface crack may be caused by

\footnotetext{
${ }^{1}$ The Centre for Advanced Robotics @ Queen Mary (ARQ), School of Electronic Engineering and Computer Science, Queen Mary University of London, London, United Kingdom

${ }^{2}$ Department of Bioengineering, Imperial College of Science, Technology and Medicine; London, United Kingdom.

${ }^{3}$ Robotics Research, Office of the CTO, Ocado Technology, United Kingdom

$\dagger\{$ f.palermo,i.farkhatdinov $\} @$ qmul.ac.uk
}

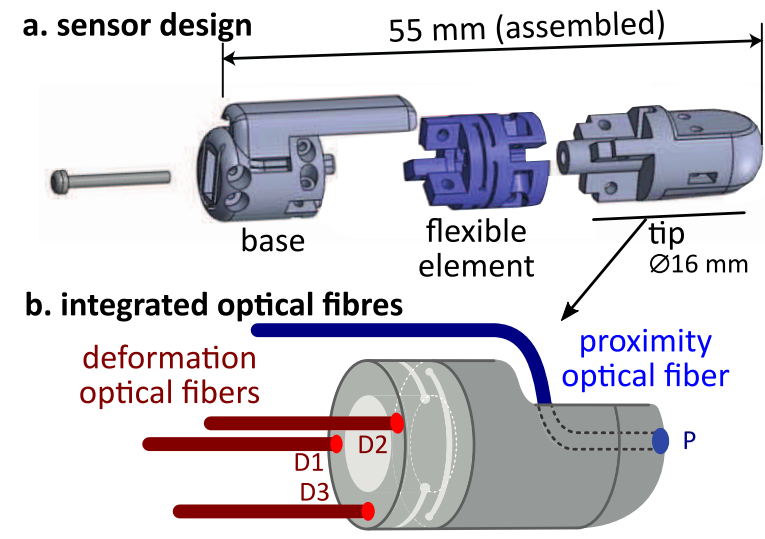

Fig. 1. Hybrid fibre optical force/proximity fingertip sensor: a. schematic design of the main mechanical components; b. the tip of the sensor with integrated fibre optics based sensing elements. Elements D1, D2, D3 indicate the three pair of fibre optics which respond to the deformation of the softer middle part of the sensor. $\mathrm{P}$ corresponds to the proximity fibre optical sensing element.

incorrect design or environment changes (e.g., temperature or pressure). The effects of undetected fractures may lead to larger macro-scale catastrophic failures making the cracked surface vulnerable to strength loss.

Existing techniques for crack detection rely on the visual analysis of the analysed segment [10], the implementation of eddy current [11], in the case of metallic structures, or ultrasonic techniques [12]. Chen et al. [13] propose a fusion between a convolutional neural network and a Naive Bayes to analyse video frames for crack detection in nuclear reactors. The proposed framework achieves a $98.3 \%$ hit rate against 0.1 false positives per frame. Schmugge et al. [14] suggest an offline crack detection method for nuclear power plant inspection video recordings by fine-tuning a deep neural network for detecting local patches containing cracks which are then grouped in spatial-temporal space for group-level classification which obtains an increase of $40 \%$ in the F1Score with respect to the compared methods. Liliopolus et al. [15] analyse the evolution of a cracking concrete structure obtained by applying digital image correlation, acoustic emission and ultrasonic pulse velocity techniques. The results highlight the time of onset and location of crack initiation as well as the width and depth of the cracks.

As described above existing crack detection methods are based on computer vision techniques and can fail in remote environments with limited luminosity. Furthermore, visionbased methods are not capable of acquiring material properties such as texture and hardness. In contrast to the visual 
modality, tactile and proximity sensing can provide important information on material properties such as shape, texture and hardness [16], [17]. Tactile sensors were efficiently used to characterise different materials in robotic teleoperation. Liu et al. [18] developed a 6-axis force/torque finger-shaped sensor capable of estimating the instantaneous friction force and normal force to recognize physical properties of the surface of unknown objects. An average classification accuracy of $88.5 \%$ is obtained when implementing a naïve Bayes classifier on 12 different textures. A multimodal tactile sensor and human-like exploration strategies has been used by Wong et al. [19] to characterise geometry of a robot's environment including curvature and dimensions. An expanded tactile sensors module has been implemented for rubber stamps inscribed with alphabets letter recognition [20]. The stiffness of objects has been investigated [21], [22] implementing a hybrid force and proximity finger-shaped sensor achieving $87 \%$ classification accuracy on a set of household objects with different stiffness values. An optical sensor has been implemented by Huang et al. [23] to detect target objects in dynamic environments prior to contact allowing the teleoperator to feel the object without an actual contact improving the benefits of touch interaction to the operator, without negative consequences of the robot contacting unknown geometrical structures. Surprisingly, not many approaches use tactile sensing for crack detection and characterisation.

Present work demonstrates how tactile and proximity sensing can be efficiently used to perform automatic crack detection. The proposed method applies machine learning techniques to detect cracks and bumps based on the deformation and proximity signals which are recorded during physical interaction between a custom-designed robotic finger and the remote environment. In case a crack is detected, the proposed automated technique measure its width. A fibre optic sensor has been implemented for data acquisition due to its compact dimensions $(\sim 55 \mathrm{~mm})$, weight $(\sim 200 \mathrm{~g})$, low cost, the strong immunity to electromagnetic interference and the the improved environmental resistance. This approach may be implemented also in extreme environments (e.g. in nuclear plants), since gamma radiation does not interfere with the basic sensing mechanism of fibre optic-based sensors [24]. To the best of the authors' knowledge, this is one of the first works on crack recognition based on hybrid fibre optical force and proximity sensor.

The paper is organized as follows. The proposed crack detection method is introduced in section II. Section III describes the experimental methodology of the work. Section IV introduces the results of the investigation. Section V presents the conclusions.

\section{CRACK DETECTION With TACTILE SENSING}

\section{A. Tactile and proximity sensor}

In this work, an integrated force and proximity fingershaped sensor described in [21] is used for automatic crack detection. The sensor is shown in Figure 1. The sensor is made of 3D printed rigid (VeroClear Glossy) and flexible (Nylon) components allowing it to bend during interaction

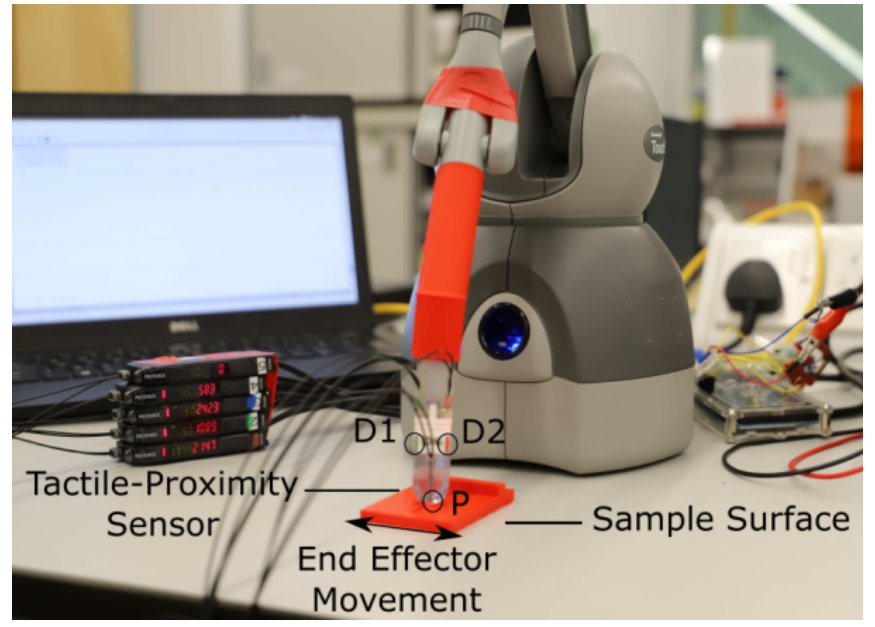

Fig. 2. The experimental setup for the data acquisition composed of fibre optics based sensor attached to desktop robotic interface (Touch/Phantom Omni), Keyence fibre optic transducers and a sample object.

with the environment. The sensor employs three pairs of optical fibre cables (D1, D2, D3) to measure the sensor's body deformation of the flexible middle part based on the changes in reflected light intensity. The sensor is capable of measuring bending torque and normal contact force during physical interaction with the environment. The fourth pair of optical fibre cables $(\mathrm{P})$ is used to sense the proximity between external objects and the tip of the finger.

The sensor's fibre optic cables are attached to an optoelectronic system to convert light intensities into voltage signals. In this work, Keyence FS-N11MN light-to-voltage transducers ${ }^{1}$ are implemented. Thus, the change of light intensity modulation is measured, and, using a calibration matrix converted to force, torque and distance measurements.

\section{B. Classification algorithm}

The goal of the proposed classification algorithm is to detect and characterise mechanical fractures, such as cracks, based on the force and proximity data recorded from the sensors of section II-A. The time history of the force and proximity data is recorded and feature extraction is performed; the resultant output is to be used as an input for the classification algorithm. Feature extraction is performed on each consequent $25 \mathrm{~ms}$ long time window with an increment of $5 \mathrm{~ms}$. The size of the time window was selected based on the sampling frequency. Data are sampled at $400 \mathrm{~Hz}$ corresponding to one data sample every $2.5 \mathrm{~ms}$. Thus, feature extraction is executed on windows of 10 data points with a window shift of two data points. The window length has been chosen empirically through grid search analysis. The Mean Absolute Value (MAV), a time domain feature, was extracted as feature. The advantage of Time Domain features is that

\footnotetext{
${ }^{1}$ https: //www.keyence.com/products/sensor/ fiber-optic/fs-n/models/fs-n11mn/
} 


\section{Surface samples}

for crack recognition:

for width classification:

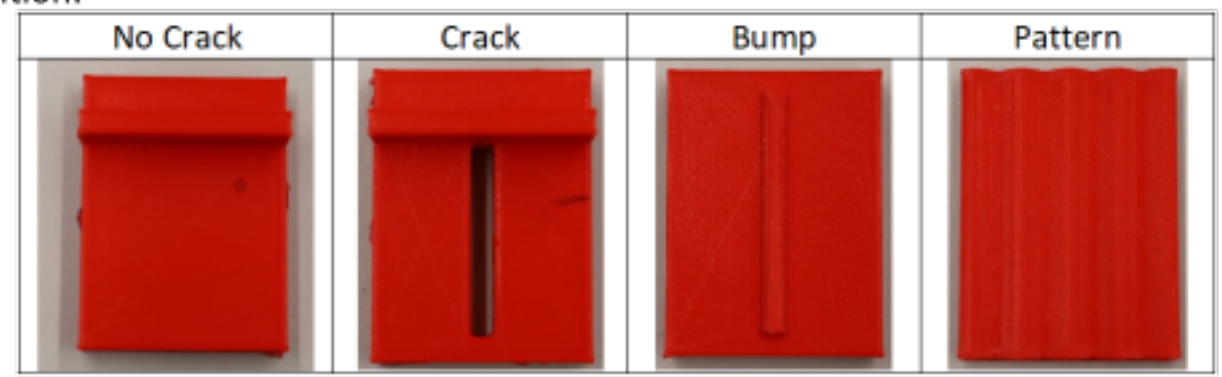

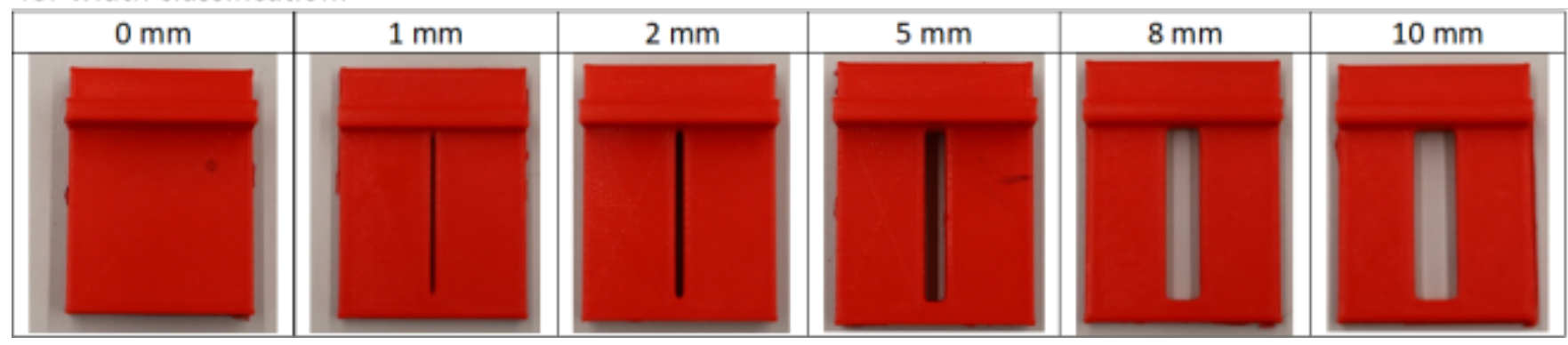

Fig. 3. Visualization of the set of objects explored during the experiments. The test set for the Crack Recognition Analysis is formed by no crack, crack, bump and wavy pattern surfaces. The series for the Crack Width classification experiment is made up of the same surface with distinct width holes of $0,1,2,5,8$ and 10 millimetres.

they are fast to calculate since they do not require any mathematical transformation. On the other hand, they are sensitive to noise. This feature has been previously implemented in surface Electromyography (sEMG) and demonstrated high performance [25], [26]. A Random Forest Classifier was used to determine both the surface pattern of examined material and the size of the detected cracks. Random Forest classifier [27] uses several classification trees to improve the classification rate. Each tree computes the analysis and the forest chooses the most voted result among the trees or the one with the highest classification accuracy. Random Forest classifier has been already evaluated for remote sensing [28] and it can successfully handle high data dimensionality since it is both fast and insensitive to overfitting. To recognize the surface of the material, the classification labels were equal to: 'no crack', 'crack', 'bump', 'wavy texture' (representing an undulating surface). Additionally, one more forest tree classifier was implemented to estimate the width of the crack once one is detected. The same data structure and Mean Absolute Value feature were used for the training of the crack width classifier. The training labels corresponded to the width of the crack in millimeters: $0,1,2,5,8,10$. Both analyses have been performed with MATLAB R2018 on a Dell Latitude 7280 laptop running on Windows 10 .

\section{EXPERIMENTAL METHODOLOGY}

\section{A. Setup}

To collect data and test the proposed crack detection algorithm, the tactile and proximity sensor, described in section
II-A, has been attached to the end-effector of a Touch desktop haptic interface (formerly known as Phantom Omni) as shown in Figure 2. The Phantom Omni was programmed to slide the tactile sensor along a static sample surface following a pre-programmed periodic movement. Data from tactile and proximity sensors were recorded through an Arduino Mega ADK micro-controller at $400 \mathrm{~Hz}$ and later synchronized with the absolute position of the tip of the tactile sensor calculated through the encoder readings of the Phantom Omni. Data acquisition and control were implemented through dedicated software libraries (OpenHaptics and Robotic Operating System) running on an Ubuntu desktop computer. The material samples, as well as the Phantom Omni interface, were statically fixed to a laboratory desk to minimise any vibration and unwanted displacements.

\section{B. Data acquisition protocol}

In this work, two experiments were conducted: crack detection and crack width classification. A set of 10 objects with different surfaces (no crack, cracks of different widths, a bump and a wavy pattern) were manufactured employing 3D printing technology (Ultimaker III ${ }^{2}, 0.2 \mathrm{~mm}$ layer height). The wavy pattern consists of a repeated pattern of waves of $1 \mathrm{~mm}$ amplitude and $5 \mathrm{~mm}$ magnitude. The samples are shown in Figure 3. Each type of these sample objects corresponds to a label used in the classifiers. The Phantom Omni moved the tactile sensor across the sample objects: the periodic sliding has a magnitude of $1.6 \mathrm{~cm}$ and a frequency of $1000 \mathrm{~Hz}$. The

\footnotetext{
${ }^{2}$ https://ultimaker.com/3d-printers/ultimaker-3
} 
average sliding velocity was $3.89 \mathrm{~mm} / \mathrm{s}$. The initial position of the tactile sensors was not controlled and varied from trial to trial at approximately 5-10 $\mathrm{mm}$ from the crack edge. No normal force was applied by the sensor to the sampled surfaces except the force caused by the sensor's weight. For both classifiers, tactile and proximity signals from 12 repeated continuous sliding movements were recorded. This continuous recording was reiterated five times.

\section{Data Analysis}

Figure 4 shows a sample of raw data acquired on 'no crack', 'crack', 'bump' and 'wavy surface' for a continuous recording. Mean Absolute Value (MAV) feature extraction was performed for a sliding $25 \mathrm{~ms}$ window of each recorded signal. A set of six repeated continuous sliding movements was used for training the random forest classifier to detect cracks and a separate set of six recordings was used for testing the performance of the method after training. Hence, $50 \%$ of the data was used for testing. First, raw and MAV data are classified using only the proximity data $(\mathrm{P})$ or the force data (D1, D2, D3). Then, raw and MAV data with combined proximity and force data are classified. Each observation is trained on itself and tested against the rest of the set one at a time (e.g., observation 2 is trained on itself and tested against observations 1, 3, 4, and 5) for intersession investigation. In total, 20 results for each analysed feature are obtained. Kruskal-Wallis statistical analysis, which indicates if the data samples come from the same distribution, is performed on the whole set of results. The same training and testing approaches were used for the width detection classifier.

\section{RESUlTS}

Random Forest Classifier, a supervised learning algorithm, with 100 trees is implemented. First, raw and MAV data with only proximity data $(\mathrm{P})$ and only force data, from the three fibre optics pairs of cable (D1, D2, D3), are used by the classifier as the baseline. Then, raw and MAV results with both force and proximity data are evaluated. The classification algorithm has been chosen instead of regression since this work focuses on discrete labels. In the future, regression analysis will be employed and evaluated for crack width recognition.

\section{A. Crack Recognition}

The goal of the Crack Recognition experiment is to recognize the presence of a crack in the object. Figure 5 shows the complete results for the classification analysis.

From Table I, it is possible to infer that the lowest classification accuracy of $53.43 \%$ is obtained when classifying Mean Absolute Value (MAV) data with only proximity data. Whilst, the best classification accuracy of $86.43 \%$ is achieved when implementing the MAV feature for both force and proximity data. Still, using only the MAV feature, without any proximity data, allows us to obtain results that are higher than the chance level for the considered number of labels $(25 \%)$. The Kruskal-Wallis test was performed on the results
TABLE I

CLASSIFICATION ACCURACY FOR CRACK RECOGNITION

\begin{tabular}{|c|c|c|}
\hline Feature & Mean & Standard Deviation \\
\hline Raw - Proximity & $57.12 \%$ & 3.94 \\
\hline MAV - Proximity & $53.43 \%$ & 2.57 \\
\hline Raw - Force & $71.72 \%$ & 8.80 \\
\hline MAV - Force & $79.87 \%$ & 8.45 \\
\hline Raw - Force + Proximity & $81.78 \%$ & 7.33 \\
\hline MAV - Force + Proximity & $86.43 \%$ & 7.72 \\
\hline
\end{tabular}

TABLE II

CRACK WIDTH CLASSIFICATION ACCURACY

\begin{tabular}{|c|c|c|}
\hline Feature & Mean & Standard Deviation \\
\hline Raw - Proximity & $35.08 \%$ & 3.20 \\
\hline MAV - Proximity & $31.31 \%$ & 2.47 \\
\hline Raw - Force & $37.81 \%$ & 6.14 \\
\hline MAV - Force & $45.85 \%$ & 5.99 \\
\hline Raw - Force + Proximity & $48.94 \%$ & 5.45 \\
\hline MAV - Force + Proximity & $57.30 \%$ & 6.66 \\
\hline
\end{tabular}

of the classification analysis of the different features and the value obtained $(p<0.001)$ indicates that the null hypothesis of having all data samples from the same distribution is rejected. Thus, there are significant differences among the implemented features.

\section{B. Crack Width Classification}

The scope of the crack width classification experiment is to classify the width in millimetres $(\mathrm{mm})$ of the fracture of the explored object. Figure 6 shows the complete results for the classification analysis.

Table II shows that the lowest classification accuracy of $31.31 \%$ is obtained when classifying Mean Absolute Value data with only proximity data. Whilst, the best classification accuracy of $57.30 \%$ is achieved when implementing MAV feature force data together with proximity. Nevertheless, using only the MAV feature, without any proximity data, allows us to obtain results that are higher than the chance level for the considered number of labels $(\sim 16.6 \%)$. Kruskal-Wallis results $(\mathrm{p}<0.001)$ indicate statistically significant differences among results obtained when analysing only force data, only proximity data and both force and proximity data.

\section{CONClusion}

This work demonstrated how tactile and proximity sensing can be efficiently used to perform automatic crack detection. The proposed method uses machine learning techniques to detect cracks and bumps based on fibre optical proximity signals which are recorded during physical interaction between a custom-designed robotic finger and the remote environment. Experimental validation of the proposed method has shown that it is possible to achieve almost $86.46 \%$ crack detection and $57 \%$ crack width classification accuracy. Kruskal-Wallis results $(\mathrm{p}<0.001)$ indicate statistically significant differences among results obtained when analysing only force data, only proximity data and both force and proximity data.

In contrast to previous techniques, which rely on visual modality, the proposed approach based on optical fibres 
Fiber optic raw signal magnitudes

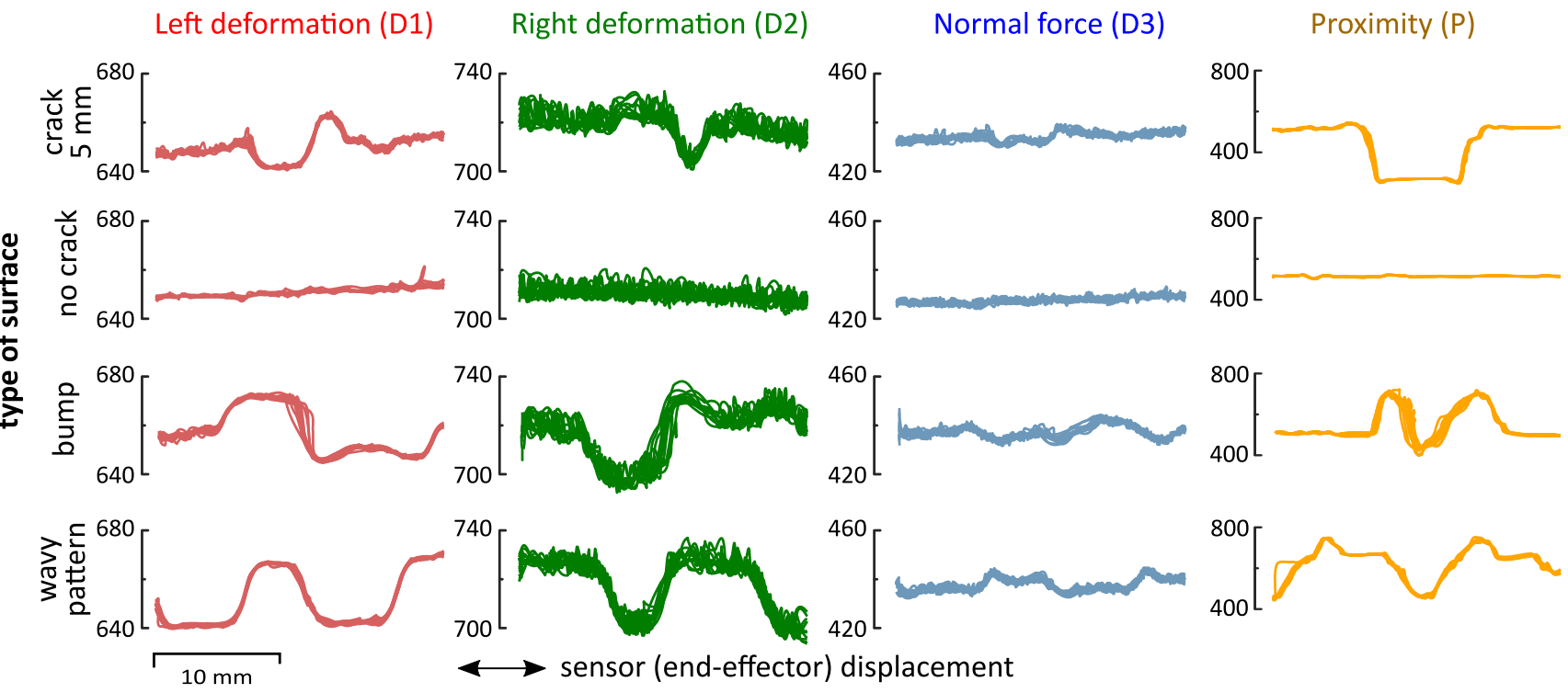

Fig. 4. Representation of raw data of the fibre optical force/proximity sensor for a set of surface samples: 'no crack', 'crack', 'bump' and 'wavy pattern'. Each column shows magnitude of the optical signal for a given optical fibre signal when the sensor was displaced on the sample surface.

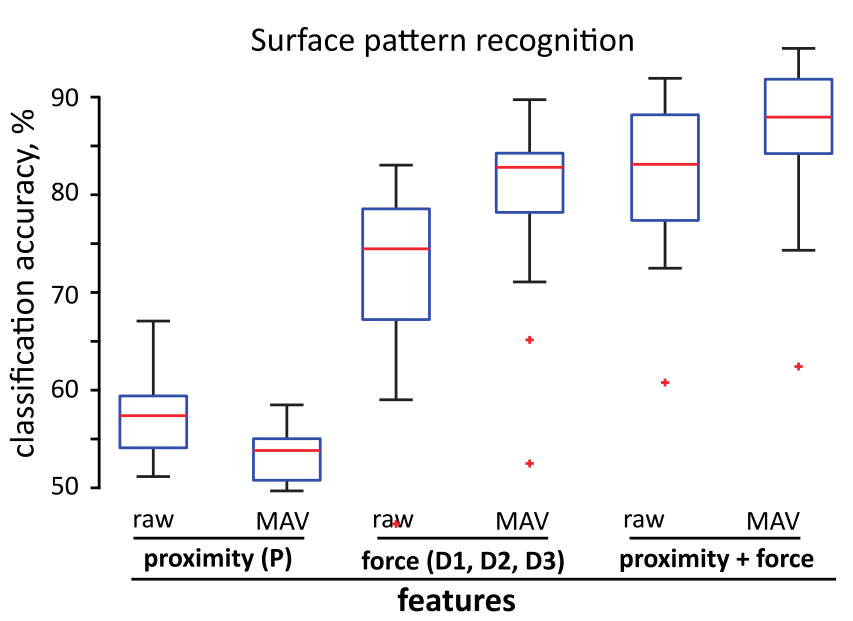

Fig. 5. Classification accuracy results for the crack recognition analysis. Raw and MAV data with only proximity data and only force data are used as baseline. Central red mark indicates the median. The bottom and top edges of the box indicate the 25 th and 75 th percentiles, respectively. The outliers are shown with the symbol '+'.

is suitable for operation in extreme environments, such as nuclear facilities where radiation damages electronic components such as video cameras.

Future research and applications will focus on a integrating multi-modal approach with visual patches and development of a real-time classifier with better accuracy to recognize the presence of a crack online and creating a $3 \mathrm{D}$ reconstruction of it on Unity.

\section{ACKNOWLEDGMENT}

The authors would like to thank the National Centre for Nuclear Robotics (UK EPSRC grant NCNR EP/R02572X/1)

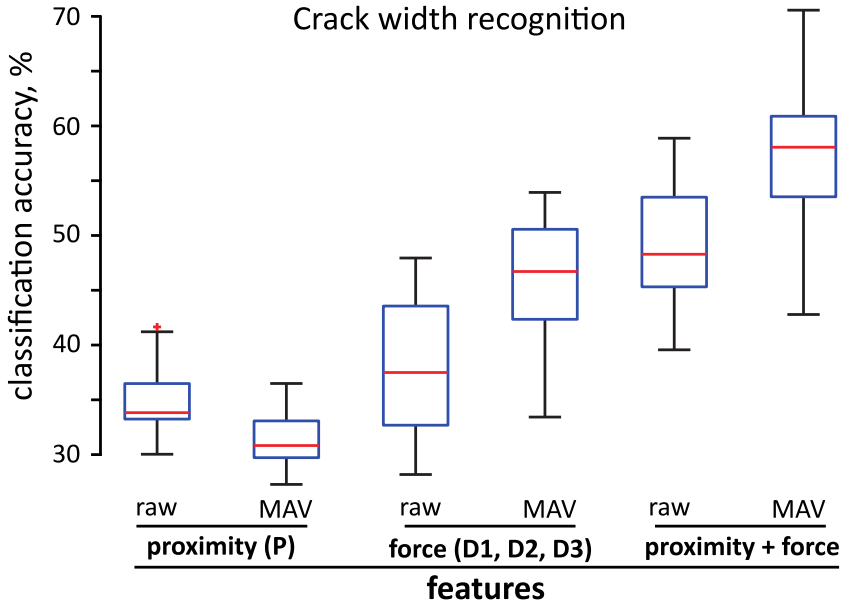

Fig. 6. Crack width classification accuracy results. Raw and MAV data with only proximity data and only force data are used as baseline. Central red mark indicates the median. The bottom and top edges of the box indicate the 25th and 75th percentiles, respectively. The outliers are shown with the symbol ' + '.

for its support of this work. Althoefer and Farkhatdinov were partially supported by the Alan Turing Institute, UK.

\section{REFERENCES}

[1] A. Shaukat, Y. Gao, J. A. Kuo, B. A. Bowen, and P. E. Mort, "Visual classification of waste material for nuclear decommissioning," Robotics and Autonomous Systems, vol. 75, pp. 365-378, 2016.

[2] L. Sun, C. Zhao, Z. Yan, P. Liu, T. Duckett, and R. Stolkin, "A novel weakly-supervised approach for rgb-d-based nuclear waste object detection," IEEE Sensors Journal, vol. 19, no. 9, pp. 3487-3500, 2018.

[3] D. W. Hainsworth, "Teleoperation user interfaces for mining robotics," Autonomous Robots, vol. 11, no. 1, pp. 19-28, 2001.

[4] M. Tavakoli, A. Aziminejad, R. V. Patel, and M. Moallem, "Highfidelity bilateral teleoperation systems and the effect of multimodal haptics," IEEE Transactions on Systems, Man, and Cybernetics, Part B (Cybernetics), vol. 37, no. 6, pp. 1512-1528, 2007. 
[5] Q. Lin and C. Kuo, "On applying virtual reality to underwater robot tele-operation and pilot training," International Journal of Virtual Reality (IJVR), vol. 5, no. 1, pp. 71-91, 2015.

[6] I. Farkhatdinov, J.-H. Ryu, and J. Poduraev, "Control strategies and feedback information in mobile robot teleoperation," IFAC Proceedings Volumes, vol. 41, no. 2, pp. 14681-14686, 2008.

[7] A. M. Okamura, "Methods for haptic feedback in teleoperated robotassisted surgery," Industrial Robot: An International Journal, vol. 31, no. 6, pp. 499-508, 2004.

[8] K. A. Manocha, N. Pernalete, and R. V. Dubey, "Variable position mapping based assistance in teleoperation for nuclear cleanup," in Robotics and Automation, 2001. Proceedings 2001 ICRA. IEEE International Conference on, vol. 1. IEEE, 2001, pp. 374-379.

[9] V. Pruks, I. Farkhatdinov, and J.-H. Ryu, "Preliminary study on realtime interactive virtual fixture generation method for shared teleoperation in unstructured environments," in International Conference on Human Haptic Sensing and Touch Enabled Computer Applications. Springer, 2018, pp. 648-659.

[10] A. Mohan and S. Poobal, "Crack detection using image processing: A critical review and analysis," Alexandria Engineering Journal, vol. 57, no. 2, pp. 787-798, 2018.

[11] Y. Yao, S.-T. E. Tung, and B. Glisic, "Crack detection and characterization techniques-an overview," Structural Control and Health Monitoring, vol. 21, no. 12, pp. 1387-1413, 2014.

[12] Y. Chang, Y. Zi, J. Zhao, Z. Yang, W. He, and H. Sun, "An adaptive sparse deconvolution method for distinguishing the overlapping echoes of ultrasonic guided waves for pipeline crack inspection," Measurement Science and Technology, vol. 28, no. 3, p. 035002, 2017.

[13] F.-C. Chen and M. R. Jahanshahi, "Nb-cnn: deep learning-based crack detection using convolutional neural network and naïve bayes data fusion," IEEE Transactions on Industrial Electronics, vol. 65, no. 5, pp. 4392-4400, 2017.

[14] S. J. Schmugge, L. Rice, N. R. Nguyen, J. Lindberg, R. Grizzi, C. Joffe, and M. C. Shin, "Detection of cracks in nuclear power plant using spatial-temporal grouping of local patches," in 2016 IEEE Winter Conference on Applications of Computer Vision (WACV). IEEE, 2016, pp. 1-7.

[15] S. Iliopoulos, D. Aggelis, L. Pyl, J. Vantomme, P. Van Marcke, E. Coppens, and L. Areias, "Detection and evaluation of cracks in the concrete buffer of the belgian nuclear waste container using combined ndt techniques," Construction and Building Materials, vol. 78, pp. 369-378, 2015.

[16] Z. Kappassov, J.-A. Corrales, and V. Perdereau, "Tactile sensing in dexterous robot hands," Robotics and Autonomous Systems, vol. 74, pp. 195-220, 2015.
[17] T. P. Tomo, S. Somlor, A. Schmitz, L. Jamone, W. Huang, H. Kristanto, and S. Sugano, "Design and characterization of a three-axis hall effectbased soft skin sensor," Sensors, vol. 16, no. 4, p. 491, 2016.

[18] H. Liu, X. Song, J. Bimbo, L. Seneviratne, and K. Althoefer, "Surface material recognition through haptic exploration using an intelligent contact sensing finger," in 2012 IEEE/RSJ International Conference on Intelligent Robots and Systems. IEEE, 2012, pp. 52-57.

[19] R. D. P. Wong, R. B. Hellman, and V. J. Santos, "Haptic exploration of fingertip-sized geometric features using a multimodal tactile sensor," in Next-Generation Robots and Systems, vol. 9116. International Society for Optics and Photonics, 2014, p. 911605.

[20] H.-K. Lee, S.-I. Chang, and E. Yoon, "A flexible polymer tactile sensor: Fabrication and modular expandability for large area deployment," Journal of microelectromechanical systems, vol. 15, no. 6, pp. 16811686,2006

[21] J. Konstantinova, G. Cotugno, A. Stilli, Y. Noh, and K. Althoefer, "Object classification using hybrid fiber optical force/proximity sensor," in 2017 IEEE SENSORS. IEEE, 2017, pp. 1-3.

[22] J. Konstantinova, A. Stilli, and K. Althoefer, "Fingertip fiber optical tactile array with two-level spring structure," Sensors, vol. 17, no. 10, p. 2337, 2017.

[23] K. Huang, P. Lancaster, J. R. Smith, and H. J. Chizeck, "Visionless tele-exploration of $3 \mathrm{~d}$ moving objects," in 2018 IEEE International Conference on Robotics and Biomimetics (ROBIO). IEEE, 2018, pp. 2238-2244.

[24] F. Berghmans, A. F. Fernandez, B. Brichard, F. Vos, M. C. Decreton, A. I. Gusarov, O. Deparis, P. Megret, M. Blondel, S. Caron et al., "Radiation hardness of fiber optic sensors for monitoring and remote handling applications in nuclear environments," in Process Monitoring with Optical Fibers and Harsh Environment Sensors, vol. 3538 International Society for Optics and Photonics, 1999, pp. 28-39.

[25] M. Hakonen, H. Piitulainen, and A. Visala, "Current state of digital signal processing in myoelectric interfaces and related applications," Biomedical Signal Processing and Control, vol. 18, pp. 334-359, 2015.

[26] F. Palermo, M. Cognolato, A. Gijsberts, H. Müller, B. Caputo, and M. Atzori, "Repeatability of grasp recognition for robotic hand prosthesis control based on semg data," in 2017 International Conference on Rehabilitation Robotics (ICORR). IEEE, 2017, pp. 1154-1159.

[27] L. Breiman, "Random forests," Machine learning, vol. 45, no. 1, pp. 5-32, 2001.

[28] M. Belgiu and L. Drăguţ, "Random forest in remote sensing: A review of applications and future directions," ISPRS Journal of Photogrammetry and Remote Sensing, vol. 114, pp. 24-31, 2016. 